\title{
A PERCEPÇÃO DE COORDENADORES, PROFESSORES E ALUNOS SOBRE A FORMAÇÃO INICIAL DE PROFESSORES DE EDUCAÇÃO FÍSICA EM DESENVOLVIMENTO INTEGRAL DA CRIANÇA
}

\author{
LA PERCEPCIÓN DE COORDINADORES, PROFESORES Y ESTUDIANTES SOBRE \\ LA FORMACIÓN INICIAL DEL PROFESORADO DE EDUCACIÓN FÍSICA EN EL \\ DESARROLLO INTEGRAL DEL NIÑO
}

\author{
THE PERCEPTION OF COORDINATORS, TEACHERS AND STUDENTS \\ ABOUT THE TEACHER INITIAL EDUCATION OF PHYSICAL EDUCATION IN THE \\ INTEGRAL DEVELOPMENT OF THE CHILD
}

\author{
Ana Patrícia Cavalcante de QUEIROZ ${ }^{1}$ \\ Carlos Alberto FERREIRA ${ }^{2}$ \\ Heraldo Simões FERREIRA ${ }^{3}$
}

RESUMO: A percepção de coordenadores, professores e alunos sobre a formação inicial de professores de educação física em desenvolvimento integral da criança, caracteriza-se como um estudo que buscou verificar as perspectivas destes atores sobre se a formação inicial proporciona a abordagem da educação física numa lógica de desenvolvimento integral da criança, nos cursos de formação inicial de professores de educação física de duas Universidades públicas do Estado do Ceará. Este estudo foi desenvolvido através de uma metodologia de investigação qualitativa, descritiva, interpretativa, concretizada pela coleta de dados realizada através de entrevista semiestruturada. Como resultado, verificamos que nas percepções da maioria dos participantes do estudo os dois cursos de formação inicial de professores de educação física abordam conhecimentos que formam e preparam para o trabalho com o desenvolvimento integral da criança. Contudo, alguns atores apontam as formações complementares e continuadas como elementos imprescindíveis para esse aperfeiçoamento profissional em desenvolvimento integral da criança.

PALAVRAS-CHAVE: Formação inicial de professores. Educação física. Desenvolvimento integral da criança. Perspectivas de atores.

RESUMEN: La percepción de los coordinadores, docentes y estudiante sobre la formación inicial de los docentes de Educación Física en el desarrollo integral del niño, se caracteriza como un estudio que buscó verificar las perspectivas de estos actores sobre si la formación inicial proporciona el abordaje de la educación fisica en una lógica de desarrollo integral

\footnotetext{
${ }^{1}$ Universidade de Trás-os-Montes e Alto Douro (UTAD), Vila Real - Portugal. Doutoranda em Ciências da Educação. ORCID: https://orcid.org/0000-0001-8026-2737. E-mail: anapatriciacq@hotmail.com

${ }^{2}$ Universidade de Trás-os-Montes e Alto Douro (UTAD), Vila Real - Portugal. Professor Auxiliar, Diretor da Licenciatura em Educação Básica e Vogal da Direção do Doutoramento em Ciências da Educação. Doutorado em Educação: especialidade em Desenvolvimento Curricular (UTDA). ORCID: https://orcid.org/0000-00020211-9780. E-mail: caferreira@utad.pt

${ }^{3}$ Universidade Estadual do Ceará (UECE), Fortaleza - CE - Brasil. Professor no Programa de Pós-Graduação em Educação. Doutorado em Saúde Coletiva (UECE). ORCID: https://orcid.org/0000-0003-1999-7982. E-mail: heraldo.simoes@uece.br
}

RIAEE - Revista Ibero-Americana de Estudos em Educação, Araraquara, v. 16, n. esp. 1, p. 1014-1028, mar. 2021. e-ISSN: 1982-5587 
del niño, en los cursos de formación inicial para maestros de Educación Física de dos Universidades públicas en el Estado de Ceará. Este estudio se desarrolló a través de una metodología de investigación cualitativa, descriptiva e interpretativa, concretada por la recopilación de datos realizada a través de una entrevista semiestructurada. Como resultado, verificamos que en las percepciones de la mayoría de los participantes del estudio, los dos cursos de formación inicial para docentes de Educación Física abordan conocimientos que forman y preparan para el trabajo con el desarrollo integral del niño. Todavía, algunos actores señalan las formaciones complementarias y continuadas como elementos esenciales para esa evolución profesional en desarrollo integral del niño.

PALABRAS CLAVE: Formación inicial del profesorado. Educación física. Desarrollo integral del niño. Perspectivas de los actores.

ABSTRACT: The perception of coordinators, teachers and students about the teacher's initial formation of the physical education in the integral development of the child, is characterized how a study that sought verify the perspectives these actors about the INITIAL formation provides the approach of the physical education in a logic of integral development of the children, in the courses OF initial formation of the teachers' physical education of the two public universities of the Ceara State. This study it was development through of a methodology of the qualitative investigation, descriptive, interpretive concretized by the data collection performed though of semi structured interview. How result it's possible verify that the participants' big perception of the study in the two courses of the initial formation of the teachers in physical education studied, the provide formation contemplate knowledge that form and prepare to job with the integral development of the child. However, some actors too point to complementary formations and continuous like important elements in the professional performance in integral development of the child.

KEYWORDS: Teachers initial education. Physical education. Integral development of the child. Perspectives of the actors.

\section{Introdução}

A formação inicial de professores se constitui através de um processo acadêmico que prepara o futuro professor a atuar de forma intelectual, educativa, pedagógica e ética no desenvolvimento e constituição dos saberes dos escolares (CUNHA, 2015; PINHO; PEIXOTO, 2017).

No tocante à formação inicial de professores de educação física, o professor deve estar preparado, ao concluir os estudos de licenciatura, a atuar de forma a proporcionar o desenvolvimento integral da criança em todas as etapas escolares (MACIEL; QUEIROZ, 2020; QUEIROZ, 2019; QUEIROZ; FERREIRA, 2017; 2018). Neste sentido, o professor de educação física escolar possui um papel significativo na promoção do desenvolvimento integral da criança e por isso deverá estar apto ao planejamento e execução de atividades, 
adequadas à faixa etária e aos níveis individuais de desenvolvimento, que promovam o desenvolvimento psicomotor, social-afetivo e cognitivo dos estudantes de forma integrada (OLIVEIRA et al., 2018; SANTOS; PRETTE; PRETTE, 2018; ZANELLA; SOUZA; VALENTINI, 2018).

Neste contexto, estabelecemos o seguinte problema de investigação: quais as percepções de coordenadores, professores e alunos dos cursos de educação física, de duas Universidades públicas do Estado do Ceará - Brasil, sobre se a formação inicial proporciona a abordagem da educação física numa lógica de desenvolvimento integral da criança?

Baseados neste problema de investigação, estabelecemos o seguinte objetivo geral para a investigação: verificar as perspectivas de coordenadores de cursos, de professores e de alunos de dois cursos de formação inicial de professores de educação física de duas universidades públicas do Estado do Ceará sobre se a formação inicial proporciona a abordagem da educação física numa lógica de desenvolvimento integral da criança.

A seguir, apresentamos a fundamentação teórica sobre o conceito e aprendizagens a realizar na formação inicial de professores, o conceito de desenvolvimento integral da criança e a importância da formação inicial de professores de educação física em desenvolvimento integral da criança. Na sequência apresentamos a metodologia de investigação, o problema e o objetivo, os procedimentos de recolha e de análise de dados, e as questões referentes às percepções dos coordenadores, professores e alunos sobre essa formação. Por fim, apresentamos e discutimos os dados das entrevistas e as considerações finais.

\section{Formação inicial de professores}

A formação inicial de professores consiste num período de intensas aprendizagens de saberes e de competências profissionais, através da associação de conhecimentos préexistente a novos conhecimentos, necessárias para atuar no ambiente escolar de forma a atender aos desafios atuais da docência (CUNHA, 2015; SILVA; CRUZ, 2017).

Ferreira (2017, p. 264) aponta que o currículo de um curso de formação inicial de professores deve se constituir através da integração dos diferentes componentes de aprendizagem e constituição profissional, pois somente desta forma "é que os futuros professores poderão realizar aprendizagens significativas dos diferentes saberes e adquirirem as competências profissionais necessárias à docência”.

A escola, ambiente de atuação deste futuro profissional, constitui-se como um espaço de vivência e criação de novas necessidades e aprendizagens que estimulem o 
desenvolvimento integral dos estudantes, deste o seu desenvolvimento pessoal, e a adaptação da criança à complexidade social (SILVA; CRUZ, 2017).

Os cursos de formação inicial de professores devem estruturar seu currículo de forma a integrar os diferentes componentes da formação e a atender às necessidades dos alunos que frequentam a escola, que, consequentemente, atende às necessidades da sociedade. Neste sentido, essa formação inicial deve promover a aquisição de conhecimentos e o desenvolvimento de competências do saber/fazer docente, que possibilitem que o futuro professor exerça a docência com conhecimento científico, curricular, didático-pedagógico, organizacional e ético (CUNHA, 2015; SILVA; CRUZ, 2017).

\section{Desenvolvimento integral da criança}

O desenvolvimento integral da criança consiste na complexa relação entre a aquisição e o desenvolvimento de habilidades e aptidões (físicas, motoras, cognitivas e psicossociais) que se adquire ao longo da vida e que são estimulados por processos científícos e não científicos (PESSOA et al., 2017).

Durante o processo de desenvolvimento integral, a criança passa por diversas transformações que estão diretamente relacionadas à sua constituição biológica, às suas características hereditárias e por mudanças adquiridas e moldadas através da sua interação social e com o conhecimento histórico da sociedade humana (CORDERO et al., 2019).

Cordero et al. (2019), afirma que o processo de desenvolvimento integral da criança, relacionado com a sua interação com o meio ambiente, se evidencia em quatro elementos: o contexto, que compreende a família e os ambientes com os quais a criança vai compartilhar suas experiências de aprendizagem e desenvolvimento; o processo, que se constitui nas vivências e interações relacionadas com as necessidades de desenvolvimento; a pessoa, que evidencia que o desenvolvimento é um processo único de cada ser; e o tempo, que ressalta que cada ser se desenvolve em seu tempo.

Assim, podemos perceber que o desenvolvimento integral da criança se caracteriza como um processo contínuo e dinâmico de influência mútua dos conjuntos funcionais (afetivo, motor e cognitivo), no qual a conquista/evolução de uma etapa de desenvolvimento é caracterizada pelo domínio de uma etapa anterior (ARCÓN; SÁNCHEZ, 2018; LUZ et al., 2017).

Neste sentido, reforça-se a importância do papel do professor no desenvolvimento integral do aluno, pois a criança deve aprender e vivenciar conhecimentos que promovam o 
seu desenvolvimento pessoal (enquanto ser único) e social (que necessita conviver e interagir socialmente) (ARCÓN; SÁNCHEZ, 2018; LUZ et al., 2017).

\section{Formação inicial de professores de educação física em desenvolvimento integral da criança}

No Brasil, os principais documentos legislativos educacionais (Lei de Diretrizes e Bases (LDB); Diretrizes Curriculares Nacionais (DCN) para a educação básica e ensino superior; os Parâmetros e Referenciais Curriculares elaborados pelo Ministério da Educação; as orientações do Conselho Nacional de Educação; e as Diretrizes Curriculares Nacionais para os cursos de graduação, licenciatura) sinalizam que a escola deve promover o desenvolvimento integral dos alunos e recomendam que os cursos de formação inicial de educação física devem preparar os futuros professores para atuar na educação básica de forma a garantir o desenvolvimento integral de todos os alunos (PONTES, 2017; QUEIROZ, 2019; QUEIROZ; FERREIRA, 2017; 2018).

A disciplina de educação física escolar trabalha constantemente a relação do indivíduo com seu corpo, na relação consigo (desenvolvimento da autoconfiança e do aprender a conhecer e a dominar seus corpos) e com os outros. Assim, o professor desta disciplina será um elemento de extrema importância no ambiente escolar para a promoção do desenvolvimento integral dos escolares (BAGNARA; FENSTERSEIFER, 2019a; 2019b; QUEIROZ; SANTOS; PRETTE; PRETTE, 2018).

Para atender aos objetivos do ambiente educacional na promoção do desenvolvimento integral dos alunos, o professor de educação física deve obter uma formação profissional que o capacite para atuar de forma a garantir esse desenvolvimento integral (QUEIROZ, 2020; ROCHA; NASCIMENTO, 2017).

Neste sentido, a formação inicial de professores de educação física deve ofertar conhecimentos profissionais adequados e necessários para que o futuro professor seja capaz de planejar, executar e avaliar atividades que atendam às necessidades reais de cada aluno, de acordo com a faixa etária e o nível de desenvolvimento, para que estes consigam alcançar, efetivamente, o seu desenvolvimento integral (TRIANI; MAGALHÃES; NOVIKOFF, 2017; ZANELLA; SOUZA; VALENTINI, 2018).

Assim, a formação inicial de professores de educação física é consolidada através do processo de aquisição de conhecimentos e de competências profissionais, pessoais e sociais, que capacitam o futuro professor para intervir no ambiente escolar de modo a promover o 
desenvolvimento integral de seus alunos (BAGNARA; FENSTERSEIFER, 2019a; 2019b; QUEIROZ; FERREIRA, 2017).

\section{Metodologia}

Considerando o problema e o objetivo deste estudo pareceu-nos adequada a utilização de uma metodologia de investigação qualitativa, com característica descritiva, interpretativa e fenomenológica (LAKATOS; MARCONI, 2017). Utilizando como cenário o curso de formação inicial de professores de educação física na modalidade licenciatura de duas universidades do Estado do Ceará, esta pesquisa se estruturou no modelo de estudo exploratório de dois casos (LAKATOS; MARCONI, 2017). Ressaltamos que este artigo caracteriza-se como um recorte de um estudo mais amplo sobre formação de professores de educação física, mas que neste momento, para este estudo, vamos dar ênfase às percepções sobre a formação de professores de educação física em desenvolvimento integral da criança dos seguintes atores que participaram no estudo: 1 coordenador; 4 professores e 6 alunos de cada um dos cursos estudados. Para acedermos às percepções dos referidos atores realizamos uma entrevista semiestruturada a cada ator. Os dados recolhidos foram submetidos à análise de conteúdo (BARDIN, 2016), da qual emergiram as seguintes categorias e subcategorias: Coordenadores, categorias (a formação em desenvolvimento integral da criança) e subcategorias (contributo do currículo para a atuação em desenvolvimento integral da criança; e perspectivas futuras); Professores, categorias (a formação em desenvolvimento integral da criança), e subcategorias (contributo do currículo para a atuação em desenvolvimento integral da criança); e Alunos, categorias (preparação para atuar no desenvolvimento integral da criança), e subcategorias (apto; formação complementar durante a formação inicial; formação complementar após a graduação; contribuição dos docentes).

\section{Percepção de coordenadores sobre a formação em desenvolvimento integral da criança}

Os coordenadores dos cursos de formação inicial de professores de educação física participantes do estudo foram questionados sobre se consideram que a formação proporcionada no curso que coordena prepara os futuros professores para atuar com o desenvolvimento integral da criança. Os coordenadores responderam: 
Eu, particularmente, acho que nossos alunos têm conseguido trabalhar bem nisso, tanto pela essa organização curricular geral, quanto os estágios, acho que os estágios têm ajudado muito (Coordenador Universidade 1).

Então, eu não acho que nossos profissionais estejam capacitados para o desenvolvimento integral da criança em etapas da educação básica. [...] eles estão mais bem preparados do que antes [...]. Mas, acredito que daqui alguns anos a gente vai conseguir ter um curso específico pra formação integral da criança (Coordenador Universidade 2).

$\mathrm{Na}$ percepção do coordenador do curso da Universidade I, o currículo do curso contribui para que o futuro professor seja capaz de atuar com o desenvolvimento integral da criança. Segundo Queiroz e Ferreira $(2017 ; 2018)$, o currículo de um curso de formação inicial de professores possui relação direta com a aprendizagem do futuro profissional no exercício de sua docência para a promoção do desenvolvimento integral.

Ainda podemos observar que o coordenador 1 faz referência aos estágios como disciplinas importantes na formação do futuro professor em relação ao desenvolvimento integral da criança. Segundo Mendes (2016) o estágio se caracteriza como uma disciplina curricular obrigatória para a conclusão dos cursos de formação inicial de professores e constitui um momento da formação no qual o futuro professor vivencia a profissão, adquirindo saberes e competências profissionais docentes.

$\mathrm{Na}$ percepção do coordenador do curso de educação física da Universidade II, o currículo não prepara os alunos futuros professores para atuarem numa perspectiva de desenvolvimento integral da criança. Ressaltamos que no Brasil o curso de graduação em licenciatura tem como objetivo capacitar o futuro professor para a lecionação de sua disciplina em todas as etapas da educação básica, que vai desde o ensino infantil até o ensino médio e que é responsável por promover o desenvolvimento integral dos estudantes no ambiente escolar (MACIEL; QUEIROZ, 2020; PONTES, 2017; QUEIROZ; FERREIRA, 2017; 2018).

O coordenador da Universidade II também afirma que, apesar de não ser a ideal, atualmente essa formação dos futuros professores de educação física já apresenta melhoras sob a ótica do desenvolvimento integral da criança. E evidencia que possui perspectivas de que no futuro o curso que coordena possa ofertar um currículo com mais ênfase no desenvolvimento de competências para a promoção do desenvolvimento integral da criança. 


\section{Percepção dos docentes sobre o contributo do currículo dos cursos na formação em desenvolvimento integral da criança}

Em relação às percepções dos docentes sobre o contributo do currículo do curso de formação inicial de professores de educação física na formação dos futuros professores no desenvolvimento integral da criança, afirmam o seguinte:

A contribuição consiste principalmente na ênfase ao processo reflexivo acerca da ambiguidade epistemológica-conceitual e teórico-metodológica que permeia a noção de desenvolvimento integral, de modo que os (as) estudantes elaborem uma postura rigorosa, sensivel e (auto) crítica na sua prática educativa como professores (as) (Professor 1 - Universidade I).

[...] há indicios de algumas disciplinas no curso que zelam pelo olhar nessa perspectiva integral de uma criança plena (Professor 2 - Universidade I).

A contribuição é muito relevante, pois trabalham conteúdos que identificam áreas biopsicossociais da criança e como trabalhar de forma a contribuir com o seu melhor desenvolvimento, identificando fases especificas, metodologias de trabalho adequadas, experiências práticas sobre esse grupo etc. (Professor 4 - Universidade II).

Os professores 1 e 2 da Universidade I e o professor 4 da Universidade II evidenciam contributos eficazes e reais do currículo do curso de formação inicial de professores de educação física para a formação dos futuros professores no desenvolvimento integral da criança. Essas percepções estão de acordo com todos os documentos oficiais educacionais que referem que a escola, e consequentemente o professor, desempenha um papel significativo na promoção do desenvolvimento pleno do aluno no ambiente escolar (BAHIA, 2016; QUEIROZ, 2019).

A percepção do professor 1 também indica que o currículo do curso da Universidade I promove uma convergência de saberes contextualizados que proporciona a formação do aluno de forma crítica/reflexiva, facilitando a compreensão e formação do futuro professor para atuar no ambiente escolar, promovendo o desenvolvimento integral da criança. Essa percepção está de acordo com e as orientações do Conselho Nacional de Educação que afirmam que os cursos de formação inicial de professores devem promover uma formação acadêmico-profissional crítica/reflexiva, fundamentada na congruência de saberes para a reflexão, compreensão e construção da prática pedagógica (FRANCO; LONGAREZI; MARCO, 2019; QUEIROZ, 2019; QUEIROZ; FERREIRA, 2017; 2018).

Para o professor 4 da Universidade II, o currículo do curso de formação inicial de professores de educação física contribui para a formação em desenvolvimento integral da criança e apresenta como elementos significativos desta formação os conteúdos das 
disciplinas, as atividades e práticas pedagógicas desenvolvidas pelo corpo docente. Esta percepção vai ao encontro de Queiroz (2020), e Rocha e Nascimento (2017) quando afirmam que a estruturação e execução do currículo são essenciais para que se oportunize uma formação do futuro professor para o exercício da docência na promoção da educação integral da criança.

Ainda analisando esse excerto narrativo o professor 4 da Universidade II também relaciona o desenvolvimento da criança com o conhecimento sobre as áreas 'biopsicossociais' da criança. Tal é referido por Luz et al. (2017), Pessoa et al. (2017), Arcón e Sánchez (2018), que afirmam que a promoção do desenvolvimento integral está diretamente relacionada com o desenvolvimento da criança enquanto ser constituído através das transformações biológicas, naturais e da interação social.

Ressaltamos que os demais docentes participantes da pesquisa não contribuíram com respostas a este item.

\section{Percepção dos alunos sobre a formação em desenvolvimento integral da criança}

Quando questionados os alunos dois cursos de formação inicial de professores sobre se consideram que a formação que obtêm no curso que frequentam os capacitam para lecionar a educação física numa perspectiva de desenvolvimento integral da criança, responderam o seguinte:

Não. Pois, precisaria me aprofundar mais no assunto de um modo geral (Aluno 1 - Universidade I).

Sim. Pois, a base que temos nesses quatro anos na universidade, sobre habilidades e capacidades motoras, que é o que eu entendo sobre desenvolvimento integral, é suficiente (Aluno 2 - Universidade I).

Sim. Apesar das deficiências da grade curricular. Pois, temos quatro estágios onde passamos por cada etapa da educação básica [...] (Aluno 3 Universidade I).

Sim. Pois, a minha formação deu subsidios suficientes (Aluno 4 Universidade I).

Sim. Mas, também acredito que preciso continuar estudando, para entender mais esse desenvolvimento integral (Aluno 5 - Universidade I).

Bem, eu me sinto capacitado pra isso, mas não só por conta da universidade. A universidade teve um papel muito importante, mas paralelo a isso busquei outras formas de aprendizagem. [...] trabalhei no ensino infantil e no ensino fundamental, então esse estágio, ele me deu uma

RIAEE - Revista Ibero-Americana de Estudos em Educação, Araraquara, v. 16, n. esp. 1, p. 1014-1028, mar. 2021. e-ISSN: 1982-5587 
bagagem muito boa para trabalhar com criança. A gente vai aprendendo algumas teorias e vai observando em alguns lugares cotidianos, é o conhecimento que a gente adquiriu aqui, pondo em prática (Aluno 6 Universidade I).

Mas, em relação ao desenvolvimento, a gente não tem como garantir: há, eu sou capacitada! Não! [...] Você está em construção da sua formação para trabalhar com essas crianças. [...], mas, cabe a mim, ou qualquer outro profissional, ou qualquer outro aluno da graduação, buscar mais saberes (Aluno 1- Universidade II).

Eu me considero, sim. [...] Porque dentro do meu curso de graduação, eu pude participar de todos os estágios supervisionados, a gente acaba passando por todas as etapas de ensino, desde o ensino infantil até o ensino médio. E, além disso, eu fui bolsista do PIBID, então eu pude estar durante um ano, ativa, tendo um supervisor para me orientar. [...] muito das vezes, a gente tem buscado outro tipo formação (Aluno 2 - Universidade II).

Creio eu, que preciso realmente de um aprofundamento, porque a gente ver de um modo geral. A partir do momento que eu concluir a graduação, vou me especializar, vou estudar (Aluno 3 - Universidade II).

Sim! Levando em conta o que lhe falei aí, que é para trabalhar habilidades e as capacidades, então sim (Aluno 4 - Universidade II).

Acho que não, cem por cento, né! Mas, acredito que o curso deu uma base bastante boa, que eu me sinto preparada para tentar contribuir com o desenvolvimento da criança. [...] E o curso também estimula muito, a universidade em si, a você continuar procurando saberes (Aluno 5 Universidade II).

Sim, hoje eu me considero. Felizmente, eu tive ótimos professores, com ampla bagagem de conhecimento, e que contribuiram muito na minha formação (Aluno 6 - Universidade II).

Na percepção dos alunos 2, 3, 4, 5 e 6 da Universidade I e os alunos 1, 2, 3, 4, 5 e 6 da Universidade II a formação inicial proporcionada pelos cursos de formação inicial de professores de educação física os capacita para trabalhar no ambiente escolar promovendo o desenvolvimento integral da criança. Esta percepção está de acordo com Queiroz (2019) que referem que os cursos de formação inicial de professores de educação física devem promover uma formação profissional que capacite o futuro professor de educação para a docência de forma a garantir esse desenvolvimento integral dos alunos.

Contudo, o aluno 1 da Universidade I relata que não se sente apto para o trabalho com a promoção do desenvolvimento integral da criança e ressalta que necessita ampliar a sua formação profissional para atuar neste sentido. Cruz (2017, p.1168) indica um possível motivo que pode justificar o fato de os futuros professores não se sentirem capazes para a docência nas escolas: 
Em geral, prevalece a constatação de que a formação docente sofre uma carência no que se refere à preparação prática, sobressaindo a falta de articulação entre as disciplinas de formação especifica e a formação pedagógica.

A necessidade de ampliar o seu conhecimento profissional sobre a promoção do desenvolvimento integral da criança relatado pelos alunos 1 e 2 da Universidade I, também é mencionado pelos alunos 1, 3, 5 e 6 da Universidade II. Mesmo considerando-se aptos para a lecionação da educação física em desenvolvimento integral da criança, esses futuros professores enfatizam a importância da formação continuada para o aperfeiçoamento de sua prática profissional em relação a esta temática. Essa formação continuada é mencionada por Cunha (2015) e Bahia (2016) como formação complementar à formação inicial, que contribui para a complementação de conhecimentos e de competências profissionais que a formação inicial não consegue proporcionar.

O aluno 6 da Universidade I e o aluno 2 da Universidade II, indicam a formação complementar (estágios e vivências pessoais) que adquiriram durante a sua formação acadêmica como um dos motivos que proporciona o sentimento de estar apto para o trabalho com o desenvolvimento integral da criança. Ressaltamos que as atividades de formação complementar devem ser ofertadas pelos cursos e instituições de ensino com o objetivo de qualificar a formação dos profissionais e que estas atividades são asseguradas pela legislação brasileira (FRANCO; LONGAREZI; MARCO, 2019; QUEIROZ, 2019).

Em relação à percepção sobre a importância da formação complementar ofertada durante a formação inicial, o aluno 2 da Universidade II refere a importância do Programa Institucional de Bolsa de Iniciação à Docência (PIBID) para a sua capacitação ao trabalho docente no desenvolvimento integral da criança. O PIBID é um programa do governo federal que oferta bolsas a alunos dos cursos de licenciatura, com o intuito de promover aos estudantes horas extracurriculares de estágio em escolas públicas, proporcionando ampliação da vivência com o cotidiano escolar para melhor compreensão da inter-relação entre teoria e prática profissional (MENDES, 2016; TRIANI; MAGALHÃES; NOVIKOFF, 2017).

O aluno 2 da Universidade II também evidencia a importância do acompanhamento e da orientação do docente do curso de formação inicial de professores durante a sua experiência no PIBID para a aquisição de conhecimentos sobre o desenvolvimento integral da criança. Os alunos 5 e 6 da Universidade II relataram a importância do acompanhamento e orientação ofertados pela Universidade e pelos docentes do curso de formação inicial de professores de educação física sobre o estímulo para que os futuros professores procurem 
aperfeiçoar a sua formação inicial através de atividades complementares e de formação continuada. Esta evidência do estímulo, acompanhamento e orientação da Universidade e dos docentes é referida por Cunha (2015) e Bahia (2016), pois sugerem que, durante a formação inicial de professores, as atividades formativas que colaboram para o aperfeiçoamento da prática docente devem ser ofertadas e estimuladas.

\section{Considerações finais}

As percepções dos coordenadores, professores e alunos da Universidade I evidenciam que a formação inicial de professores de educação física desta instituição proporciona uma abordagem da educação física numa lógica de desenvolvimento integral da criança. E referem que esse conhecimento profissional é constituído pela proposição de atividades que estimulam a formação crítica/reflexiva, congregando a mobilização de saberes teóricos com a prática profissional contextualizada. As percepções dos professores e dos alunos da Universidade II indicam que o curso de formação inicial de professores de educação física desta instituição contribui para a formação em desenvolvimento integral da criança e apresentam como elementos significativos os conteúdos das disciplinas e práticas pedagógicas. Porém, a percepção do coordenador deste curso da Universidade II diverge das percepções dos professores e dos alunos, pois, acredita que, por mais que já tenham ocorrido transformações e melhorias no currículo, atualmente, os alunos deste curso não estão preparados para lecionarem numa lógica de desenvolvimento integral da criança devido a lacunas na sua formação inicial. Este coordenador também deixa evidente que possui expectativas de que no futuro o curso de formação inicial de professores de educação física possa ter um currículo mais voltado para o desenvolvimento de competências para a promoção do desenvolvimento integral da criança, ou até que exista um curso específico de formação de professores relacionado com esta temática. Os alunos de ambos os cursos também referem que as formações complementares e as formações continuadas são elementos importantes para o aperfeiçoamento profissional em desenvolvimento integral da criança.

\section{REFERÊNCIAS}

ARCÓN, V. A. B.; SÁNCHEZ, A. R. R. Salud mental como efecto del desarrollo psicoafectivo en la infância. Psicogente, v. 21, n. 39, p. 183-202, jan./jun. 2018. ISSN 01240137. DOI: http://doi.org/10.17081/psico.21.39.2830 
BAGNARA, I. C.; FENSTERSEIFER, P. E. Relação entre formação inicial e ação docente: o desafio político da educação física escolar no centro do debate. Revista Brasileira de Ciências do Esporte, v. 41, n. 3, p. 277-283, jul./set. 2019a. Disponível em: http://www.rbceonline.org.br/en-estadisticas-S0101328918300751. Acesso em: 11 jun. 2020.

BAGNARA, I. C.; FENSTERSEIFER, P. E. Responsabilidade da educação física escolar: concepções dos professores que atuam na formação inicial. Revista Brasileira de Ciências do Esporte, jan. 2019b. Disponível em: https://www.sciencedirect.com/science/article/pii/S0101328918303196. Acesso em: 11 jun. 2020.

BAHIA, C. S. Formação continuada em exercício de professores de educação física escolar: contribuições para a prática-pedagógica. 2016. 194 f. Tese (Doutorado em Educação Física) - Universidade Federal de Santa Catarina, Florianópolis, Brasil, 2016. Disponível em: https://repositorio.ufsc.br/xmlui/handle/123456789/174907. Acesso em: 11 jun. 2020.

BARDIN, L. Análise de conteúdo. Trad. Reto, L. A. e Pinheiro, A. São Paulo: Edições 70, 2016. (Obra original publicada 1977)

CORDERO, K. S. et al. Teste costa-riquenho: avaliação do desenvolvimento integral da menina e do menino de 0 meses a 6 anos (EDIN II). Revista Enfermería Actual, n. 37, jul./dez. 2019. Disponível em:

https://revistas.ucr.ac.cr/index.php/enfermeria/article/view/34959/39163. Acesso em: 11 jun. 2020 .

CRUZ, G. B. Ensino de didática e aprendizagem da docência na formação inicial de professores. Cadernos de Pesquisa, v. 47, n. 166, p. 1166-1195, 2017. DOI: https://dx.doi.org/10.1590/198053144323

CUNHA, A. C. Ser professor: bases de uma sistematização teórica. Chapecó: Argos, 2015.

FERREIRA, C. A. Os (des)arranjos curriculares na formação inicial portuguesa de educadores de infância e de professores dos $1^{\circ}$ e $2^{\circ}$ ciclos do ensino básico. E-Curriculum, v. 15, n. 2, p. 253-273, abr./jun. 2017. DOI: 10.23925/1809-3876.2017v15i2p253-273

FRANCO, P. L. J.; LONGAREZI, A. M.; MARCO, F. F. A unidade teórico-prática da intervenção didático-formativa no contexto da docência. Ciência \& Educação, Bauru, v. 25, n. 3, p. 705-723, out. 2019. DOI: https://dx.doi.org/10.1590/1516-731320190030002

LAKATOS, E. M.; MARCONI, M. A. Metodologia científica. 8. ed. São Paulo: Atlas, 2017.

LUZ, C. M. N. et al. The evaluation of motor competence in typically developing children: an integrative review. Journal of Physical Education, v. 28, n. 2857, 2017. Disponível em: http://www.scielo.br/readcube/epdf.php?doi=10.4025/jphyseduc.v28i1.2857\&pid=S244824552017000100205\&pdf_path=jpe/v28/2448-2455-jpe-28-e2857.pdf\&lang=en. Acesso em: 11 jun. 2020.

MACIEL, F. A. S.; QUEIROZ, A. P. C. Direitos e conquistas na prática docente: a formação de professores universitários no Brasil. Série Educar Formação e Prática Docente. Belo Horizonte, MG: Poisson, 2020. v. 18, p. 100. 
MENDES, D. S. O estágio na licenciatura em educação física em perspectiva semiótica: (re)ver-se e (re)criar-se em imagens. 2016. 256 f. Tese (Doutorado em Educação) Universidade de São Paulo, Presidente Prudente, 2016. Disponível em: https://repositorio.unesp.br/handle/11449/141476. Acesso em: 11 jun. 2020.

OLIVEIRA, A. C. et al. Habilidades auditivas, de linguagem, motoras e sociais no desenvolvimento infantil: uma proposta de triagem. Revista CEFAC, v. 20, n. 2, mar./abr. 2018. DOI: $10.1590 / 1982-0216201820216617$

PESSOA, C. T. et al. Concepções de educadores infantis sobre aprendizagem e desenvolvimento: análise pela psicologia histórico-cultural. Psicologia Escolar e Educacional, v. 21, n. 2, 2017. DOI: http://dx.doi.org/10.1590/2175-3539/2017/02121093

PINHO, M. J.; PEIXOTO, E. R. B. A educação integral diante do novo paradigma: perspectivas e desafios. Educação e linguagens, v. 6, n. 10, jan./jun. 2017. Disponível em: http://www.fecilcam.br/revista/index.php/educacaoelinguagens/article/viewFile/1293/990. Acesso em: 11 jun. 2020.

PONTES, J. A. F. (Org.). Conhecimentos do professor de educação física escolar. Fortaleza: EdUECE, 2017.

QUEIROZ, A. P. C. A Formação inicial de professores de educação física em desenvolvimento integral da criança: um olhar sobre as orientações curriculares formais.

Revista de Estudos Curriculares, v. 10, p. 1, 2019. Disponível em: https://www.nonio.uminho.pt/rec/index.php/rec/issue/view/9/showToc. Acesso em: 11 jun. 2020 .

QUEIROZ, A. P. C.; FERREIRA, C. A. A. S. A formação inicial de professores de educação física em desenvolvimento integral da criança: a perspectiva da legislação brasileira. In: CONGRESSO DE CIÊNCIAS DO DESPORTO E DE EDUCAÇÃOO FÍSICA DOS PAÍSES DE LÍNGUA PORTUGUESA, 17., 2018, Fortaleza. Anais [...]. Fortaleza, CE: Universidade Estadual do Ceará, 2018.

QUEIROZ, A. P. C.; FERREIRA, C. A. S. A formação de professores de educação física em desenvolvimento da criança em duas universidades do Estado do Ceará. In: CATUNDA, R.; SANTOS, A. L.; AGUIAR, I. B.; FRANCHI, K. M.; PONTES JR., J. A. (Org.). Atividade física e promoção de saúde na escola: coletânea de estudos. Fortaleza: EdUECE, 2017. v. 1, p. 94-109.

ROCHA, J. C. S.; NASCIMENTO, J. V. (Re)investimento na integralidade da formação do licenciado em educação física. Revista Brasileira de Ciências do Esporte, v. 39, n. 1, p. 5662, jan./mar. 2017. Disponível em:

https://www.sciencedirect.com/science/article/pii/S0101328915001390?via\%3Dihub. Acesso em: 11 jun. 2020.

SANTOS, J. V.; PRETTE, Z. A. P. D.; PRETTE, A. D. Habilidades sociais educativas: revisão sistemática da produção brasileira. Avances en Psicología Latinoamericana, v. 36, n. 1, p. 45-63, 2018. Disponível em: http://www.scielo.org.co/pdf/apl/v36n1/1794-4724-apl36-01-00045.pdf. Acesso em: 11 jun. 2020. 
SILVA, O. B. S.; CRUZ, F. C. P. Saberes docente e formação profissional: riscos da proletarização do trabalho docente. Iniciação \& formação docente, v. 4, 2017. ISSN: 23591064.

TRIANI, F. S.; MAGALHÃES, C. A. O.; NOVIKOFF, C. As representações sociais de estudantes de educação física sobre a formação de professores. Movimento, v. 23, n. 2, p. 575-586, abr./jun. 2017. Disponível em:

http://www.seer.ufrgs.br/index.php/Movimento/article/view/68898/42071. Acesso em: 11 jun. 2020.

ZANELLA, L. W.; SOUZA, M. S.; VALENTINI, N. C. Variables that may explain motor performance changes in children with development coordination disorder and typical development. Journal of Physical Education, v. 29, maio 2018. Disponível em: http://www.scielo.br/scielo.php?script=sci_arttext\&pid=S2448-

24552018000100104\&lang=pt. doi: 10.4025/jphyseduc.v29i1.2905. Acesso em: 11 jun. 2020.

\section{Como referenciar este artigo}

QUEIROZ, A. P. C.; FERREIRA, C. A.; FERREIRA, H. S. A percepção de coordenadores, professores e alunos sobre a formação inicial de professores de educação física no aspecto ao desenvolvimento integral da criança. Revista Ibero-Americana de Estudos em Educação, Araraquara, v. 16, n. esp. 1, p. 1014-1028, mar. 2021. e-ISSN: 1982-5587. DOI: https://doi.org/10.21723/riaee.v16iEsp.1.13813

Submissão em: 20/06/2020

Revisões requeridas em: 16/09/2020

Aceito em: 03/11/2020

Publicado em: 01/03/2021 